\title{
EL APRENDIZAJE BASADO EN PROBLEMAS COMO HERRAMIENTA PARA EL ESTUDIO DELOS FENÓMENOS GEOLÓGICOS
}

Problem-Based Learning as a tool for the study of geological phenomena Aprendizagem Baseada em Problemas como uma ferramenta para estudar os fenômenos geológicos

\section{Fernando José Pereira García (1)}

\section{María Iluminada Muñoz Lucas (2)}

(1) Área de Química Analítica, departamento de Química y Física Aplicadas, Universidad de León, España. Teléfono: +34 987291535. Correo electrónico: fjperg@unileon.es.

(2) Departamento Científico-Tecnológico. Centro de Educación de Adultos Celtiberia, Soria, España. Teléfono: +34 691347534. Correo electrónico: minmaculada.mun@educa.jcyl.es

\section{Resumen}

Para suscitar interés y motivar al alumnado de $4^{\circ}$ de ESO de la materia de Biología y Geología, se ha diseñado una experiencia de enseñanza-aprendizaje fundamentada en una metodología de Aprendizaje Basado en Problemas (ABP). En este ABP se estudian las relaciones que existen, de manera no siempre evidente, entre los fenómenos geológicos y sus consecuencias biológicas, así como los procesos de causa-efecto presentes en la naturaleza. De este modo, se pretende mejorar el pensamiento crítico y abstracto de los estudiantes. La evaluación de esta buena práctica se ha realizado mediante una matriz de rúbrica, elaborada por el profesor y entregada previamente a los alumnos. Además, se ha incluido un cuestionario de evaluación de la actividad por parte de los estudiantes, resultando muy bien valorada por los mismos.

Palabras clave: Aprendizaje Basado en Problemas; Biología y Geología; vulcanismo; matriz de rúbrica; experiencia educativa

\footnotetext{
Abstract

To motivate and improve the interest of students of Biology and Geology in 4th year of ESO, a teaching-learning experience based on a Problem-Based Learning methodology 
(PBL) has been designed. In this PBL, the relationships, not always evidently, between geological phenomena and their biological consequences, as well as the cause-effect processes present in nature, are studied. In this way, it is intended to improve students' critical and abstract thinking. The evaluation of this experience has been done through a rubric, elaborated by the teacher, and given previously to the students. In addition, a questionnaire has been included to evaluate this activity by the students, being very well valued by them.

Keywords: Problem-Based Learning; Biology and Geology; volcanism; rubric; educative experience

\section{Resumo}

Para motivar e aumentar o interesse dos alunos na $4^{\text {a }}$ ESO no campo da Biologia e Geologia, foi projetada uma experiência de aprendizagem com base em uma metodologia de aprendizagem baseada em problemas (ABP). Neste ABP os relacionamentos, nem sempre óbvias, entre os fenômenos geológicos e as suas consequências biológicas e os processos de causa e efeito na natureza são estudadas. Assim, pretende-se melhorar o pensamento crítico e abstrato dos alunos. A avaliação desta boa prática foi realizada utilizando uma rubrica desenvolvida pelo professor, e previamente entregue a os alunos. Além disso, incluiu um questionário de avaliação dos alunos, resultando altamente valorizado.

Palavras-chave: Aprendizagem Baseado em Problemas; Biologia e Geologia; vulcanismo; rubrica; experiência educacional

\section{Introducción}

Desde hace algunas décadas, el interés por el estudio de las ciencias ha ido disminuyendo (Solbes, Montserrat, \& Furió, 2007), lo que podría repercutir en el número de futuros científicos. Se han descrito diversas causas asociadas a esta desmotivación, como es el desequilibrio entre la parte teórica de las ciencias y la realidad cotidiana del alumnado (González-Carmona, 2010).

Con el fin de minimizar esta desmotivación, convendría emplear metodologías innovadoras, como el Aprendizaje Basado en Problemas (ABP). A través de él se ejercitan las Inteligencias Múltiples (Gardner, 1983) y se consolidan las competencias El Aprendizaje Basado en Problemas como herramienta para el estudio de los fenómenos geológicos 
clave, trabajando para resolver situaciones problemáticas reales (Vizcarro \& Juárez, 2008). De este modo, se fomenta la participación activa en el aula, las relaciones entre iguales y la autonomía e iniciativa personal (Caamaño, 2004; García de la Vega, 2012).

Los objetivos de esta experiencia educativa titulada La Plaga de Justiniano son promover el aprendizaje crítico y activo en el estudiante, desarrollar su pensamiento abstracto y potenciar el trabajo en grupo, mejorando la organización y la coordinación de tareas.

\section{Contextualización}

La actividad ha sido realizada por alumnos de Biología y Geología de $4^{\circ}$ de ESO de un instituto público de la ciudad de León. Se trabajó con un grupo de veinticinco estudiantes con edades entre 15 y 17 años. Estos estudiantes, cuyo rendimiento académico se puede considerar medio, no habían llevado a cabo experiencias de ABP previas.

Esta actividad se engloba en el Bloque 2 La dinámica de la Tierra, recogido en

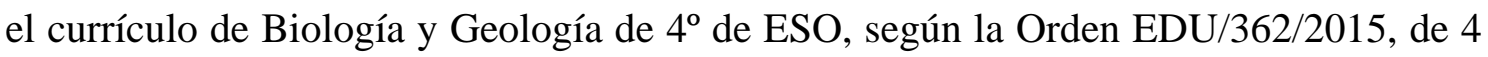
de mayo, por la que se establece el currículo y se regula la implantación, evaluación y desarrollo de la Educación Secundaria Obligatoria en la Comunidad de Castilla y León.

\section{Diseño y desarrollo}

En este ABP se propuso un trabajo de abstracción por parte de los estudiantes, consistente en determinar las posibles causas de la Plaga de Justiniano (540-541 d.C.). Para ello, se utilizaron dos sesiones al finalizar la unidad correspondiente.

En la primera sesión, el docente entregó a los alumnos un texto que recoge las crónicas de varios historiadores acerca de las nieblas persistentes, malas cosechas y hambrunas que asolaron prácticamente toda Europa en el siglo VI. También hace referencia a la epidemia de peste que, coetáneamente, se extendió por todo el Mediterráneo, causando la muerte de millones de personas. Una vez leído el texto, se resolvieron las dudas de vocabulario, comprobando que todos los alumnos lo habían entendido.

Los estudiantes se dividieron en grupos de cinco y, a continuación, cada equipo investigó cuál pudo ser la causa original de esta epidemia, proponiendo sus hipótesis, discutiendo sobre ellas y desechando las menos probables. Para ello dispusieron de El Aprendizaje Basado en Problemas como herramienta para el estudio de los fenómenos geológicos 
material de consulta, como libros y artículos científicos y periodísticos. Las ideas se anotaron en un documento que recogió el docente al concluir la clase.

En la segunda sesión, se les devolvieron sus producciones y comenzó el debate intergrupal, durante el cual los alumnos trataron de explicar sus hipótesis y rebatir las de sus compañeros. Este debate concluyó con la entrega, por parte del profesor, de otro texto, en el que se expone que, según estudios recientes, la gran actividad volcánica de América Central en el siglo VI, está directamente vinculada con un ciclo de malas cosechas en Europa, que favoreció la epidemia de peste. Los estudiantes, relacionando ambos textos, habrían dado con la respuesta a la cuestión planteada.

El producto final consistió en un informe grupal que incluía las hipótesis barajadas, las preguntas planteadas, cómo alcanzaron la solución final y el razonamiento acerca de la relación entre las causas y las consecuencias del problema.

\section{Evaluación}

Esta experiencia se evaluó empleando una matriz de rúbrica, elaborada por el profesor (Anexo 1). Esta matriz, que contiene ítems relativos al trabajo individual y al grupal, se entregó a los estudiantes en la primera sesión, con el objetivo de hacerlos partícipes de los aspectos que el docente iba a tener en cuenta a la hora de valorarlos.

La información que permitió evaluar los ítems, así como otros aspectos significativos que tuvieron lugar durante la experiencia, se recogieron en el diario del profesor.

Para evaluar la actividad, al concluir el ABP los estudiantes recibieron un cuestionario de preguntas cerradas (puntúa de 1 a 5 el interés de la actividad, las herramientas facilitadas, el tiempo disponible, etc.) y abiertas (¿cómo te has sentido trabajando en equipo?, ¿crees que es útil la realización de este tipo de actividades y por qué?, ¿qué aspectos cambiarías/mejorarías y cómo?, etc.).

\section{Conclusiones}

Desde la perspectiva del docente, esta experiencia ha resultado enriquecedora para los estudiantes en cuanto a que han sido capaces de coordinarse en grupo, formular respuestas creativas y coherentes y participar activamente en los debates. El profesor ha constituido una herramienta de apoyo, motivando y realizando preguntas clave para atraer a los alumnos hacia el ABP. 
Los alumnos se han mostrado muy interesados en la actividad, dato que se ha extraído de los cuestionarios. Esta motivación ha repercutido en su rendimiento académico, que ha mejorado con respecto al bloque curricular anterior.

Los estudiantes han trabajado las competencias básicas en ciencia y tecnología, aprender a aprender, comunicación lingüística y sentido de iniciativa y espíritu emprendedor. Se pusieron en funcionamiento, además, las Inteligencias Múltiples naturalista, inter e intrapersonal y lingüística.

Como propuesta de mejora, se plantea colaborar interdisciplinarmente con el Departamento de Geografía e Historia, ya que este ABP contiene aspectos relacionados con estas materias.

Por otra parte, y como perspectiva de desarrollo de la experiencia, se podría llevar a cabo con estudiantes de Biología y Geología de $3^{\circ}$ de ESO, puesto que también se adapta a su currículo convenientemente. Del mismo modo, se propone poner en práctica nuevos $\mathrm{ABP}$ en otros bloques de contenidos de la materia de Biología y Geología de $4^{\circ}$ de ESO.

\section{Referencias}

Caamaño, A. (2004). Experiencias, experimentos ilustrativos, ejercicios prácticos e investigaciones: ¿una clasificación útil de los trabajos prácticos? Alambique Didáctica de las Ciencias Experimentales, 39, 8-19.

García de la Vega, A. (2012). El aprendizaje basado en problemas en los itinerarios didácticos vinculados al patrimonio. Educación y Futuro, 27, 155-175.

Gardner, H. (1983). Frames of mind. The Theory of the Multiple intelligences. Nueva York: Basics.

González-Carmona, A. (2010). La importancia de las prácticas de laboratorio en la Biología y Geología y posibilidades para su desarrollo y evaluación. Innovación y Experiencias Educativas, 28, 1-10.

Solbes, J., Montserrat, R., \& Furió, C. (2007). El desinterés del alumnado hacia el aprendizaje de la ciencia: implicaciones en su enseñanza. Didáctica de las Ciencias Experimentales y Sociales, 21, 91-117. 
Vizcarro, C., \& Juárez, E. (2008). ¿Qué es y cómo funciona el aprendizaje basado en problemas? En J. García Sevilla (Ed.), La metodología del Aprendizaje Basado en Problemas (pp. 9-32). Barcelona: Dikasteia Universidad de Barcelona. 
Anexo 1.

Matriz de rúbrica para evaluar la experiencia de $A B P$

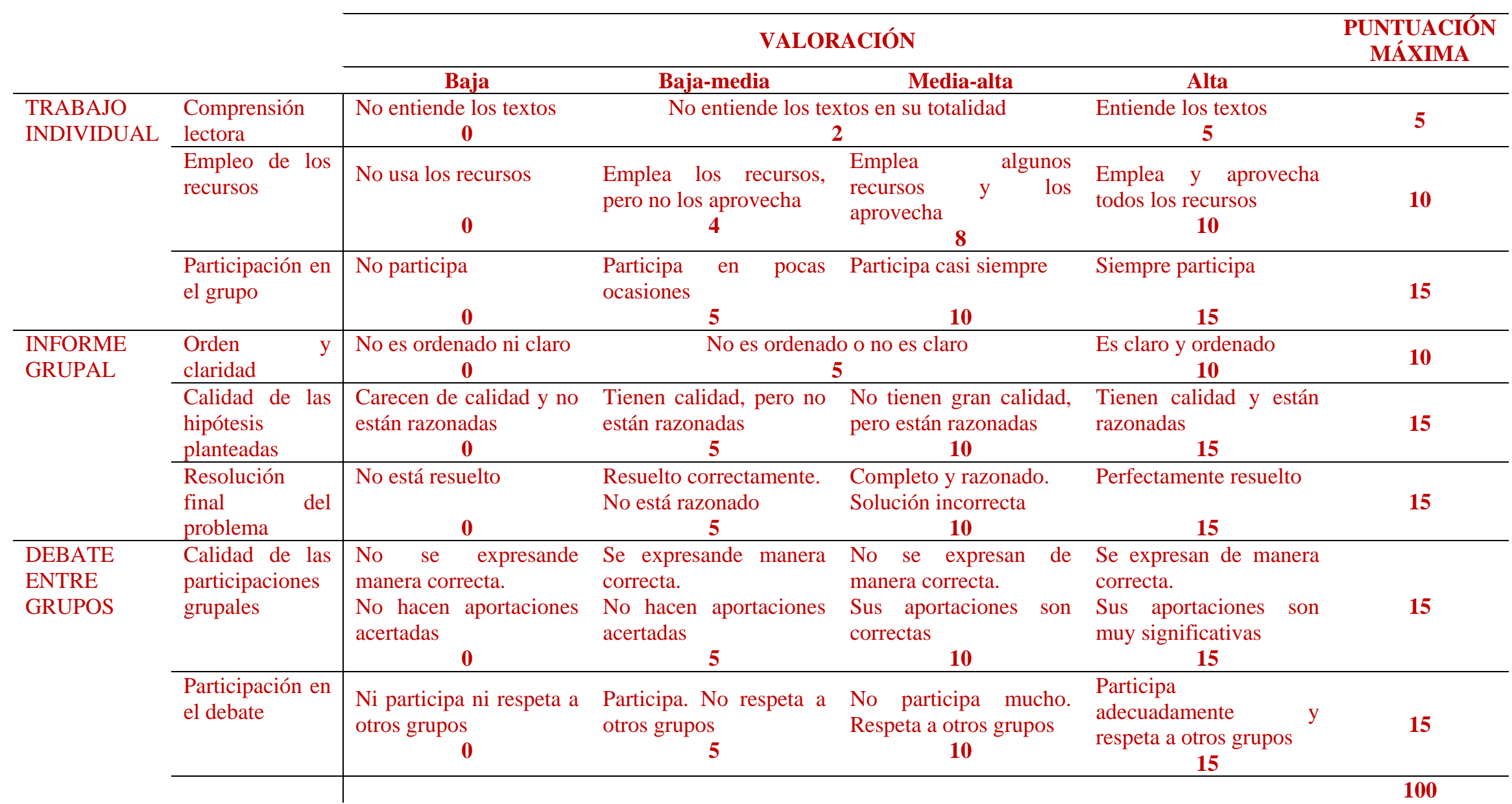

